\title{
Prediction of Concrete Pumping Using Various Rheological Models
}

\author{
Myoung Sung Choi ${ }^{1,2), *}$, Young Jin $\mathrm{Kim}^{1)}$, and Jin Keun $\mathrm{Kim}^{2)}$
}

(Received March 26, 2014, Accepted June 9, 2014, Published online July 8, 2014)

\begin{abstract}
When concrete is being transported through a pipe, the lubrication layer is formed at the interface between concrete and the pipe wall and is the major factor facilitating concrete pumping. A possible mechanism that illustrates to the formation of the layer is the shear-induced particle migration and determining the rheological parameters is a paramount factor to simulate the concrete flow in pipe. In this study, numerical simulations considering various rheological models in the shear-induced particle migration were conducted and compared with $170 \mathrm{~m}$ full-scale pumping tests. It was found that the multimodal viscosity model representing concrete as a three-phase suspension consisting of cement paste, sand and gravel can accurately simulate the lubrication layer. Moreover, considering the particle shape effects of concrete constituents with increased intrinsic viscosity can more exactly predict the pipe flow of pumped concrete.
\end{abstract}

Keywords: concrete pumping, lubrication layer, shear-induced particle migration, multimodal viscosity model, particle shape.

\section{Introduction}

Since concrete pumping was firstly introduced in 1930s, it has been a most common technique to transport fresh concrete in the real construction sites as it allows access to hard to reach region and reduces casting process duration and allows for a continuous casting. It has mostly been conducted not based on a quantitative estimation but based on hand-on experience or qualitative estimation for the pumpability through simple material tests such as slump test and bleeding test. However, a methodology for the quantitative predictions of the concrete pumping such as pumpable height or distance, pressure level, and flow rates are necessary to control the casting speed and to determine the total duration of the construction period, which is directly related to the construction cost.

Various studies (Browne and Bamforth 1977; Ede 1957; Chailmo et al. 1989; Tanigawa et al. 1991; Alekseev 1952; Weber 1968; Morinaga 1973; Sakuta et al. 1989; Jacobsen et al. 2009; Kaplan et al. 2005) have indicated that the lubrication layer which is formed at the interface between the concrete and the wall of the pipe plays a dominant role to facilitate concrete pumping (c.f. Fig. 1). Its existence was first suggested by Alekseev (1952) and Weber (1968). Morinaga (1973) also noted that, from a theoretical point of

\footnotetext{
${ }^{1)}$ Civil Engineering Research Team, Daewoo Institute of Construction Technology, Suwon 440-210, Korea.

${ }^{2)}$ Department of Civil and Environmental Engineering, Korea Advanced Institute of Science and Technology, Daejeon 305-701, Korea.

*Corresponding Author; E-mail: iamfame@kaist.ac.kr Copyright $($ The Author(s) 2014. This article is published with open access at Springerlink.com
}

view, considering the rheological parameters of the material, the pumping of concrete would not be possible without the formation of this slippage layer. Sakuta et al. (1989) went further and demonstrated that the flow properties of the bulk material were irrelevant. The only properties that matters were the ability of the material to form this layer. A study by Kaplan et al. (2005) also demonstrated that the lubrication layer is a major factor in facilitating concrete pumping because the layer has a significantly lower viscosity and yield stress than concrete as generally measured by a concrete rheometer and also developed a test instrument called a tribometer that measures the friction stress at the wall of the pipe. Kwon et al. (2013) also developed a test instrument by considering the level of the shear rate that induces the lubrication layer during real pumping. Choi et al. (2013a) tried to measure the velocity of lubrication layer for full size pumping circuits using an ultrasonic velocity profiler (UVP).

A possible mechanism that illustrates to the formation of its layer is shear-induced particle migration (Choi et al. 2013b; Phillips et al. 1992; Jo et al. 2012; Wallevik 2008; Koehler et al. 2006). When concrete is pumped, a redistribution of particles occurs near the wall of the pipe due to the gradient of the shear stress. This is a common feature of particle suspensions, and initially well-mixed particles in concentrated suspension flows undergo migration from regions of higher shear stress to those of lower shear stress. The concrete pumping can therefore be considered in most cases as the shearing of an annular layer that is a few millimeters thick and has much lower viscosity than the concrete itself. Phillips et al. (1992) verified that the shearinduced particle migration can be a formative mechanism for the formation of the layer. Choi et al. (2013b) and Jo et al. (2012) tried to simulate the formation of the layer in the concrete pipe flow with the shear-induced particle migration. 


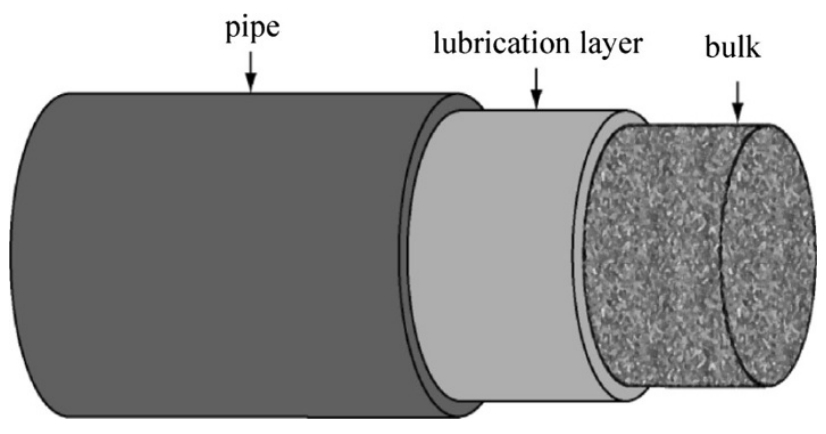

Fig. 1 Schematic pattern of concrete flow in the pipe.

When conducting the shear-induced particle migration analysis, the rheological parameters of materials are a paramount factor to predict the lubrication layer and the pumping conditions. Various rheological models (Hafid et al. 2010; Chateau and Trung 2008; Liu 2000; Krieger 1959; Chong and Baer 1971; Farris 1968) were suggested to describe the rheological behavior of suspension. However, very few studies have been performed to establish comprehensive prediction methodology for concrete pumping, that is, there have been few studies investigating the role of these rheological models on determining the pipe flow of pumped concrete, more specifically, for the formation and material properties of lubrication layer.

The objectives of this study are therefore investigating the pipe flow of pumped concrete depending on the various rheological models. The calculated results were compared with the $170 \mathrm{~m}$ full-scale pumping tests (Choi et al. 2013a). From the comparison, it was found that the multimodal suspension model representing concrete as a three-phase materials consisting of cement paste, sand and gravel with the effects of the particle shape can accurately predict the properties of lubrication layer and the pipe flow of pumped concrete.

\section{Analysis Method for the Pipe Flow of Pumped Concrete}

\subsection{Shear-Induced Particle Migration}

As stated in the introduction, to predict the pipe flow of pumped concrete, the lubrication layer should be carefully investigated. Several possible mechanisms illustrating the formation of its layer could be found in existing studies (Kaplan et al. 2005; Kwon et al. 2013; Choi et al. 2013b; Phillips et al. 1992; Jo et al. 2012; Wallevik 2008; Koehler et al. 2006). In this study, the shear-induced particle migration which demonstrated that particles in suspension migrate across the streamlines from a region of a higher shear rate to a region of a lower shear rate was considered as a major possible mechanism that contributes to the formation of the lubrication layer. Leighton et al. (Leighton and Acrivos 1987a, b) suggested phenomenological models for particle migration in non-homogeneous shear flows that typically result from spatial variation in irreversible interaction frequency and effective viscosity. Phillips et al. (1992) adapted the scaling arguments of Leighton and Acrivos (1987a, b) and proposed a diffusive flux equation to describe the time evolution of the particle concentration based on a two-body interaction model. In this study, the particle diffusive model proposed by Phillips et al. (1992), combined with general flow equations, was extended to solve the flow of concrete and predict the particle concentration distribution of suspensions in a pressure driven pipe flow.

The governing equation of the shear-induced particle migration for the Poiseuille flow is as follows (Phillips et al. 1992):

$$
\begin{aligned}
\frac{\partial \phi}{\partial t}+\frac{\partial\left(u_{z} \phi\right)}{\partial z}= & \nabla \\
& \cdot\left\{a^{2} K_{c} \phi \nabla\left(\phi \frac{\partial u_{z}}{\partial r}\right)+K_{\eta} \phi^{2} a^{2} \frac{\partial u_{z}}{\partial r} \frac{\nabla \eta}{\eta}\right\}
\end{aligned}
$$

where $\phi$ is the particle concentration, $t$ is the time, $u_{z}$ is the velocity component in the flow direction, $a$ is the particle radius, $z$ is the flow direction, $r$ is the radial direction, $\eta$ is the apparent viscosity of the concentrated suspension, and $K_{c}$ and $K_{\eta}$ are dimensionless phenomenological constants.

In the pipe flow of pumped concrete, the shear stress is the highest at the wall of the pipe and linearly decreases as the position moves to the center of the pipe. The stress gradient is a driving force to move particles toward the center of the pipe as described in the first term of the right side in Eq. (1). The increase of the particle concentration due to the migration may increase the viscosity and the yield stress, which hinder the additional migration of the particles as described in the second term of the right side in Eq. (1). As a result, the concentration of the particle inside the pipe is determined by the balance between the two actions, namely, the migration due to the stress gradient and the hindrance due to the increased rheological properties.

The governing Eq. (1) should be supplemented with appropriate boundary conditions at the pipe wall, which is subjected to the usual no slip condition $(u=0)$ and no particle flux through the wall as expressed below:

$$
\left[K_{c} \phi \nabla\left(\phi \frac{\partial u_{z}}{\partial r}\right)+K_{\eta} \phi^{2} \frac{\partial u_{z}}{\partial r} \frac{\nabla \eta}{\eta}\right] \cdot n=0
$$

The particle concentration is assumed to be uniform initially at the entrance of the pipe, i.e.

$$
\phi=\phi_{0} \quad \text { for } 0 \leq r \leq R \quad \text { at } \quad z=0
$$

where $\phi_{0}$ is the initial concentration of particles. The parameters, $K_{c}$ and $K_{\eta}$ should be independent of the particle size, concentration, and shear rate. The values of these parameters were set to 0.3 and 0.6 , respectively (Phillips et al. 1992).

\subsection{Effects of Particle Concentration and Particle Shape on Rheological Properties}

Regarding the governing equation for shear-induced particle migration in Eq. (1), the apparent viscosity $\eta$ which is a 
rheological parameter of materials should be carefully determined. The apparent viscosity $\eta$ for the Bingham fluid model can be expressed as follows:

$$
\eta=\eta_{p}+\frac{\tau_{0}}{\dot{\gamma}}
$$

where $\eta_{p}$ and $\tau_{0}$ are the plastic viscosity and yield stress of Bingham fluid model, respectively. The particle concentration variation due to particle migration might cause a considerable change in the rheological characteristics of the mixtures. For the yield stress $\tau_{0}$, one possible model that was presented in the literature was used (Hafid et al. 2010; Chateau and Trung 2008)

$$
\tau_{0}(\phi)=\tau_{0}(0) \sqrt{\frac{1-\phi_{s}}{\left(1-\phi / \phi_{s, \text { max }}\right)^{-2.5 \phi_{s, \text { max }}}}} \sqrt{\frac{1-\phi_{g}}{\left(1-\phi / \phi_{g, \text { max }}\right)^{-2.5 \phi_{g, \text { max }}}}}
$$

where $\phi_{s}$ and $\phi_{g}$ are the volume fraction of sand and gravel and $\phi_{s, \max }$ and $\phi_{g, \text { max }}$ are the maximum concentration of sand and gravel, respectively.

Through the preliminary investigation on the effects of the rheological properties on the concrete pumping (Choi et al. 2013a), the yield stress causes a minor effect on the pipe flow compared to the effects of the plastic viscosity, that is, above single yield stress model is sufficient to analyze the concrete flow. On the other hand, the plastic viscosity $\eta_{p}$ is greatly influencing on flow rates and pump pressure so that it should be carefully determined to investigate the concrete pumping.

There have been various studies to illustrate the behavior of suspensions and suggested some viscosity models (Liu 2000; Krieger 1959; Chong and Baer 1971; Farris 1968). When taking into account the concrete, suggested viscosity models can be divided into two categories depending on the scale-level of constituents: unimodal suspension model and multimodal suspension model. From a unimodal suspension point of view, concrete can be considered as a two-phase material consisting of mortar and gravel. In this approximation, mortar is the suspending medium and gravel is an only particle considered. In this study, three unimodal viscosity models were investigated.

The first unimodal viscosity model is Liu model (Liu 2000). The model is capable of predicting the viscosity of a variety of ceramic suspensions under different conditions of shear. Regarding the model, to take into account the maximum packing density, Liu proposed a model to estimate the theoretically maximum particle volume fractions $\left(\phi_{\max }\right)$ that are allowable for a given suspension at which the concrete viscosity approaches infinity. Under the Liu model, the maximum solid fraction of gravel was determined as 0.645 , for this study. The proposed viscosity model by Liu was provided as follows:

$$
\eta_{r}=\left[a\left(\phi_{\max }-\phi\right)\right]^{-2}
$$

where $a$ is a constant which was determined from the $\left(1-\eta_{r}\right)-\phi$ relationship.

The second model used in this study is the KriegerDougherty model (Krieger 1959), as follows

$$
\eta_{r}=\left(1-\frac{\phi}{\phi_{\max }}\right)^{-2.5 \phi_{\max }}
$$

This model takes into account the concentration, size distribution, and shape of the particles within the suspension. Ideally, the Krieger-Dougherty model is much better suited to evaluate the viscosity of a cement paste or concrete.

The last model was provided by Chong and Baer (1971) predicting the relative viscosity as a function of particle volume fraction and maximum packing fraction, which is defined as following:

$$
\eta_{r}=\left[1+\frac{\frac{0.75 \phi}{\phi_{\max }}}{1-\frac{\phi}{\phi_{\max }}}\right]^{2}
$$

Meanwhile, from a multimodal suspension point of view, concrete can be considered as a three-phase material consisting of cement paste, sand and gravel. Farris (1968) basically developed a theory for calculating the viscosity of multimodal suspensions of spheres. The viscosity of particles of a multimodal suspension can be calculated from the unimodal viscosity of each size as long as the relative sizes are sufficient to ensure no interaction between the gravel and sand particles. The Farris model was defined as the following:

$$
\eta_{r}=\left(1-\frac{\phi_{s}}{\phi_{s, \text { max }}}\right)^{-\left[\eta_{s}\right] \phi_{s, \text { max }}}\left(1-\frac{\phi_{g}}{\phi_{g, \text { max }}}\right)^{-\left[\eta_{g}\right] \phi_{g, \text { max }}}
$$

The Farris model breaks the aggregate parameters into two levels: sand and gravel. The volume fraction, i.e. $\phi_{s}$ and $\phi_{g}$ of each mode is determined separately according to the mix proportion and $\phi_{s, \max }$ and $\phi_{g, \text { max }}$ is the maximum solid fraction of sand and gravel, respectively. Through the Liu model (Liu 2000), the maximum solid fractions of each mode were determined as 0.675 and 0.645 , respectively, for this study condition. Where $\eta_{s}$ and $\eta_{g}$ are the intrinsic viscosity of the sand and gravel, respectively, and it represents the effects of individual particle shape on viscosity. It is defined as follows:

$$
[\eta]=\lim _{\phi \rightarrow o} \frac{\eta_{r}-1}{\phi}
$$

where $\eta_{r}$ is determined by Eq. (9). Therefore, in case of multimodal viscosity model, the effects of individual particle shape should be carefully determined in calculating the viscosity.

\subsection{Effects of Particle Shape}

Basically, Farris (1968) proposed the multimodal suspension viscosity model using a constant intrinsic viscosity value, i.e. 2.5 , for representing the spherical particles case. In 


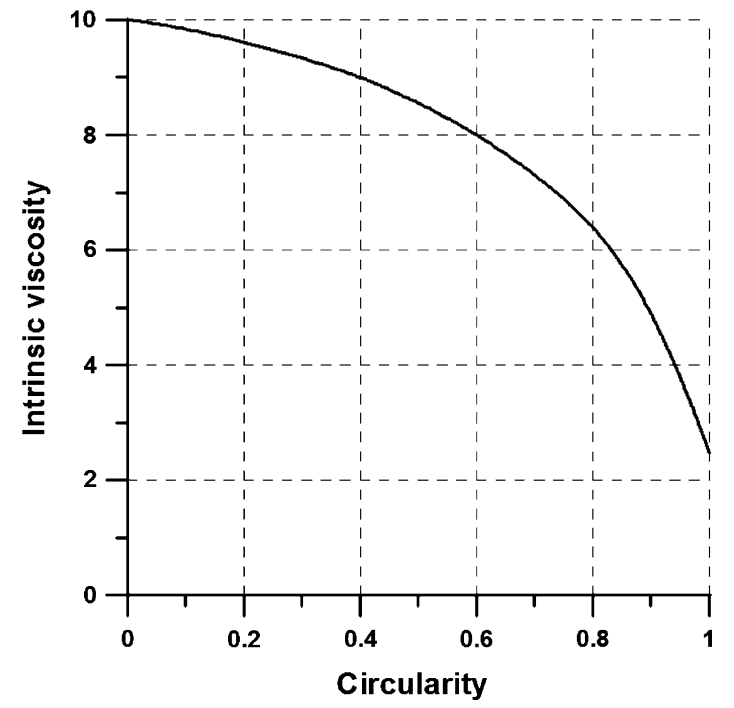

Fig. 2 Relationship between intrinsic viscosity values and circularity.

effect, the intrinsic viscosity represents the effects of the individual particle shape on the viscosity. In case of concrete, as the constituents deviate from spherical shape, modified intrinsic viscosity values should be used to simulate the effects of particle shape. According to Struble and Sun (1995) and Barnes et al. (Walters 1989), the intrinsic viscosity is 2.5 for spherical particles, and when the particles deviate from spherical shape, this value should be increased. The intrinsic viscosity is the limiting value of the reduced viscosity as the concentration of particles approaches zero. By replacing the 2.5 fraction with intrinsic viscosity, particle shapes can be considered with the expression. Therefore, to simulate the effect of particle shape on plastic viscosity, it is necessary to obtain relationship between the intrinsic viscosity and the shape characterization of the aggregates. Szecsy (1997) obtained some relationship between the intrinsic viscosity values and circularity which is a parameter to explain shape character in order to calculate the overall viscosity (c.f. Fig. 2). The maximum limit of 10 is set for the intrinsic viscosity as it is the largest reported value for intrinsic viscosity by Barnes et al. (Walters 1989). The circularity is calculated as follows:

$$
\text { Circularity }=\frac{\frac{A \times 4}{\pi}}{\left(\frac{P}{\pi}\right)^{2}}
$$

where $A$ is the cross sectional area of particle, and $P$ is the perimeter of particles. In this study, to find out the parameters of circularity, the digital image processing technique was used and more details information could be found in earlier works (Choi et al. 2013b).

\section{Experimental Programs}

\subsection{Materials and Mix Proportions}

To investigate the concrete flow in a pipe, a highlyworkable concrete (i.e. fluid concrete) was studied in this work. The mix proportion is given in Table 1. The cement was a CEM I $52.5 \mathrm{~N}$ with density $3,150 \mathrm{~kg} / \mathrm{m}^{3}$. The sand was natural river sand with density $2,590 \mathrm{~kg} / \mathrm{m}^{3}$ and fineness modulus of 2.81. Sand particle size ranges from 0.08 to $5 \mathrm{~mm}$ with water absorption capacity $2.43 \%$. The maximum coarse aggregate size was $25 \mathrm{~mm}$. It was a limestone aggregate with water absorption capacity $0.8 \%$, density $2,610 \mathrm{~kg} / \mathrm{m}^{3}$, and fineness modulus 6.72 . The amount of mixing water was corrected to take into account the water absorbed by sand and coarse aggregates. A polycarboxylatebased high range water reducing admixtures (HRWRA) was also used. Its dosage is shown in Table 1 marked as \% HRWRA, meaning the percentage of admixture relative to binder content which is the sum of cement, fly ash and blast furnace slag (in weight). The mix proportion has $4.5 \%$ air content.

\subsection{Measurement of Rheological Properties}

To determine the rheological properties of concrete by using the concentration form of particles, the rheological properties of suspension such as mortar and cement paste were measured by using a commercially available digital Brookfield rheometer equipped with cylindrical smooth spindle (cf. Fig. 3). The device keeps the temperature of the specimen constant during the entire time span of the rheological test through a water circulation system around the sample container. The test was started at the age of $15 \mathrm{~min}$ after water addition time. The tests were executed stepwise at $100,50,30,20,10$ and $5 \mathrm{rpm}$. At each rotational speed, torque and angular velocity data were recorded. The recorded results were then converted into viscosity functions such as shear stress $(\mathrm{Pa})$ and shear rate (1/s) using standard procedure (Mitschka 1982). The linear regression analysis was conducted to determine the plastic viscosity and yield stress as slope and intercept of the regression line drawn through the data points in shear stress versus shear rate plot (Lachemi et al. 2004).

\subsection{Full Scale Pumping Test}

For the experimental verification, a horizontal pumping circuit of $170 \mathrm{~m}$ length was set up (c.f. Fig. 4; Choi et al. 2013a). This circuit included eight $180^{\circ}$ and three $90^{\circ}$ bends. The pipe diameter was $125 \mathrm{~mm}$ and its thickness was $7.7 \mathrm{~mm}$. The concrete pump was a high pressure piston pump. The filling rate of the pump cylinder, which directly affects the flow rate, was calibrated from specific experiments prior to the real concrete pumping. The concrete was pumped into several $1 \mathrm{~m}^{3}$ reservoirs, which were connected to a linear variable differential transformer (LVDT). As the pump cylinder volume is known, the filling rate was computed from the LVDT length variation with designated stroke times. Through these experiments, the averaged filling rate was found to be around $85 \%$.

\subsection{Ultrasonic Velocity Profiler (UVP)}

An ultrasonic velocity profiler (UVP; Choi et al. 2013a, b; Met-flow 2002) was used to obtain a non-destructive measurement of the concrete velocity profile in the pipe using 
Table 1 Mix proportions.

\begin{tabular}{c|c}
\hline Materials & Design strength \\
\hline \hline Series name & C50 \\
\hline Cement CEM I $52.5 \mathrm{~N}\left(\mathrm{~kg} / \mathrm{m}^{3}\right)$ & 225 \\
\hline Fly ash class $\mathrm{F}\left(\mathrm{kg} / \mathrm{m}^{3}\right)$ & 50 \\
\hline Blast furnace slag $\left(\mathrm{kg} / \mathrm{m}^{3}\right)$ & 225 \\
\hline$W / B$ ratio & 0.33 \\
\hline Sand $\left(\mathrm{kg} / \mathrm{m}^{3}\right)$ & 736 \\
\hline Coarse aggregate $\left(\mathrm{kg} / \mathrm{m}^{3}\right)$ & 871 \\
\hline Polycarboxylate-based HRWRA & 0.9 \\
\hline Slump flow $(\mathrm{mm})$ & $620 \pm 20$ \\
\hline
\end{tabular}

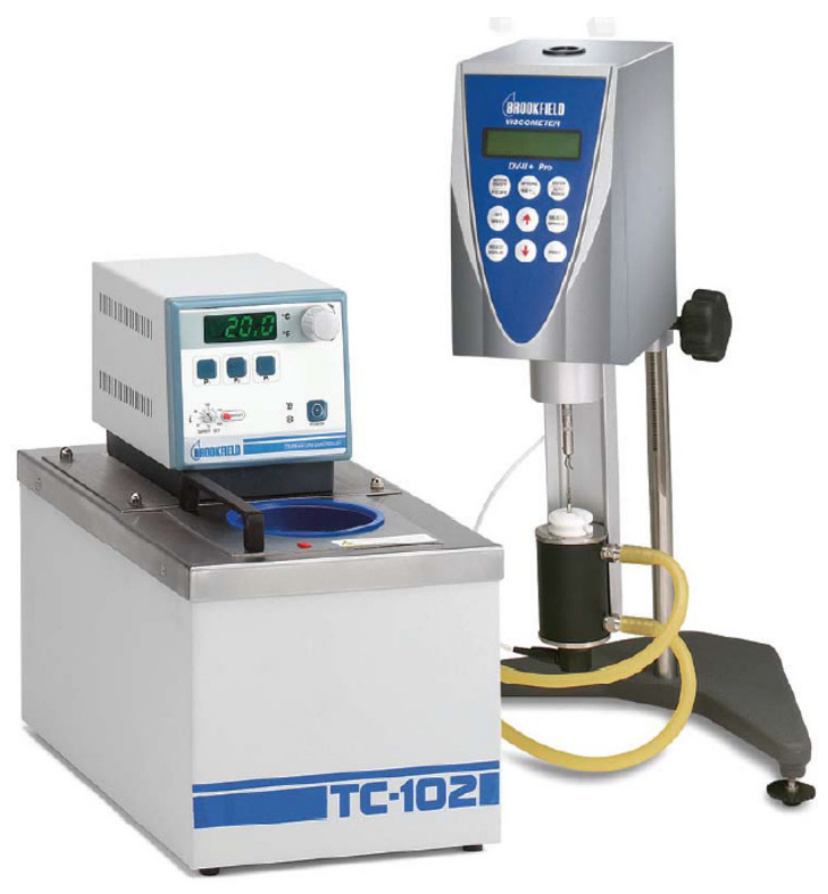

Fig. 3 Rheometer for the measurement of rheological properties of mortar and cement paste.

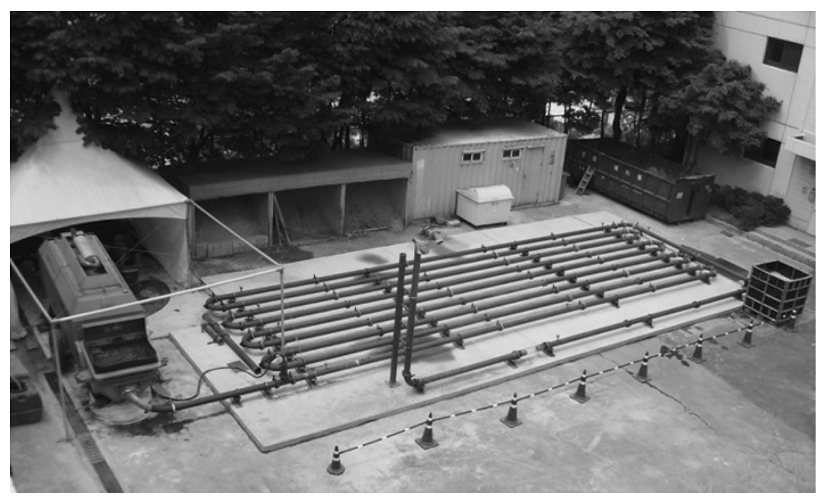

Fig. 4 Overview of the $170 \mathrm{~m}$ full-scale pumping setup.

ultrasonic waves with a high resolution. The detailed specifications of the device used in this study are given in Table 2.
The UVP allows for the measurement of the flow velocity profile using the following steps: first, there is a pulsed emission of ultrasound signals, then the echo reception and finally the detection of the Doppler shift frequency. In addition, for application of the UVP using ultrasonic waves, a $1 \mathrm{~m}$ transparent engineered plastic pipe with the same diameter as the standard pipe was installed in the last section, as shown in Fig. 5. Other information about the UVP, such as its principle of measurement, the installation of the ultrasonic probe and some limitation can be found in the earlier works (Choi et al. 2013a; Met-flow 2002).

\section{Numerical Simulation for the Pumping Test}

\subsection{Intrinsic Viscosity for Sand and Gravel}

In case of multimodal viscosity model, the modified intrinsic viscosity for sand and gravel should be determined for the shear-induced particle migration analysis. To determine an optimum intrinsic viscosity considering the particle shape effects, the circularity was quantified using digital image processing technique (Kwan et al. 1999; Mora and Kwan 2000; Otsu 1979). Through the relationship between the intrinsic viscosity and the circularity in Fig. 2, the calculated intrinsic viscosity of sand and gravel are determined as 3.5 and 6, respectively, which are the averaged values of representative samples of each aggregate which are selected randomly (Choi et al. 2013b).

\subsection{Pipe Modeling and Analysis Conditions}

Figure 6 shows the two-dimensional axisymmetric modeling of the pipe and part of the mesh. From the previous studies (Kaplan et al. 2005; Kwon et al. 2013; Choi et al. 2013a, b), the pressure distribution along the pipe length has a nearly linear relation. Therefore, for an effective numerical analysis, the $10 \mathrm{~m}$ length of the pipe was modeled for the shear-induced particle migration analysis. The detailed input values for the analysis in this study are summarized in Table 3. The commercial computational fluid dynamic (CFD) code Fluent (Fluent Inc. 2011) was used to calculate 
Table 2 Experimental UVP parameters.

\begin{tabular}{c|c}
\hline Item & Content \\
\hline Frequency $(\mathrm{MHz})$ & 8 \\
\hline Cycles per pulse & $2-32$ \\
\hline No. of profiles & 1,024 \\
\hline Sound velocity $(\mathrm{m} / \mathrm{s})$ & $2,680 \pm 200$ \\
\hline Doppler angle $\left(^{\circ}\right)$ & $85 \pm 0.5$ \\
\hline Spatial resolution $(\mathrm{mm})$ & Min. 0.20 \\
\hline
\end{tabular}

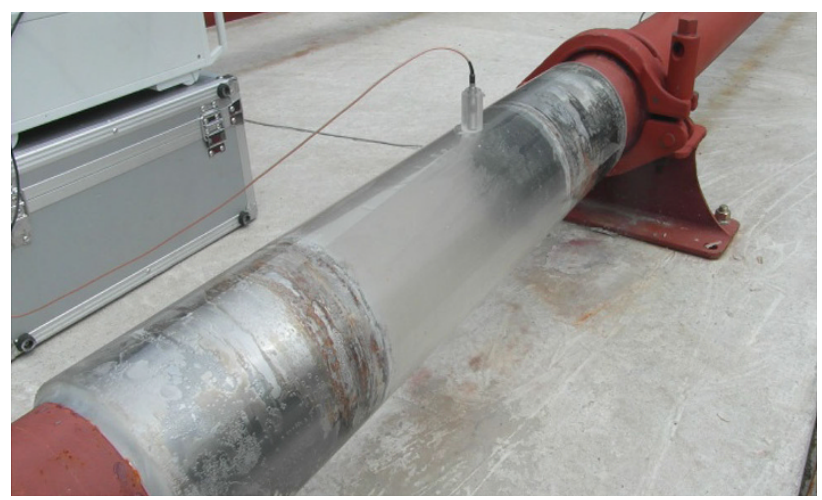

Fig. 5 Application point of the UVP and transparent engineered plastic.

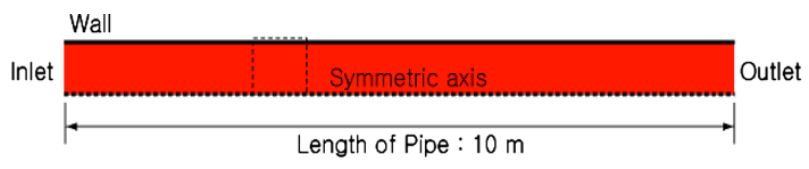

(a) Modeling of the pipe

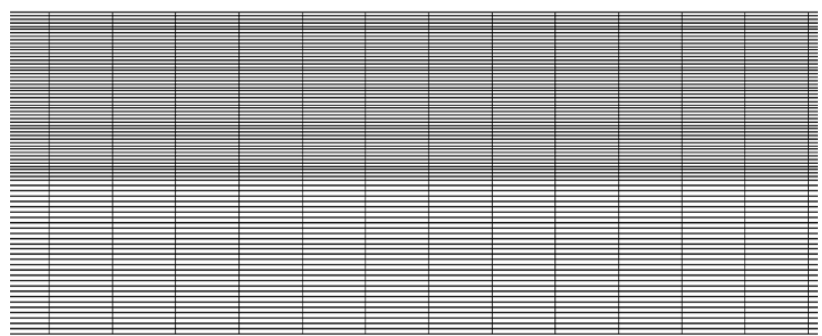

(b) Mesh section (above dashed quadrangular)

Fig. 6 Pipe modeling and mesh configuration.

the flow conditions. In addition, a subroutine program called the User Defined Scalar (Fluent Inc. 2011) was coded to solve Eq. (1) considering the four different viscosity model with particle shape effects. The subroutine program was interlocked with the general flow analysis.

\section{Results and Discussion}

\subsection{Shear Rate and Velocity}

The numerical simulations considering four different viscosity models in shear-induced particle migration analysis were conducted and then the shear rate and velocity profiles were calculated in Fig. 7. Each parameter is defined as normalized values: the shear rate and velocity are relative to the maximum shear rate and velocity, respectively, and the radius is relative to the unit length. When concerning the shear rate results, multimodal viscosity model i.e. Farris model, has steep changing near the wall but smooth changing at the center region. On the other hand, unimodal viscosity models have gradual change over the entire region, i.e. having parabolic profile. Moreover, when considering the velocity results, which were drawn by integral of shear rate, as expected, it shows similar pattern with shear rate results, that is, abrupt change near the wall for the multimodal model and parabolic change for the three unimodal models. Regarding the lubrication layer, it is a region where the shear rate and velocity are changing sharply. Thus, through these numerical calculations, it could be noted that among four viscosity models, the multimodal viscosity model can simulate more exactly the lubrication layer, which means that multimodal viscosity model is more suitable to predict the pipe flow of pumped concrete.

In order to verify the calculation results, the velocity profiles were compared with experimental results measured using UVP. The velocity was measured only approximately $20 \%$ of radius from the wall because of limitation of measurement device (Choi et al. 2013a). As shown in Fig. 8, the measured velocity also has dramatic change at the wall due to the lubrication layer which has a good agreement with calculation result using multimodal viscosity model. However, when taking a look at the profile, there is some discrepancy between the numerically calculated velocity and experimentally measured velocity. The experimental velocity profile measured by UVP has more brutal change in slope at the wall, which creates more flow rate. The discrepancy might come from the effects of particle shape. When calculating the velocity of multimodal modal, the spherical shape of particles for both modes was basically considered. To take into account the particle shape effects, the modified intrinsic viscosity should be used.

Thus, numerical simulation with increased intrinsic viscosity obtained through digital image processing was newly conducted. As shown in Fig. 9, the calculated normalized velocity with increased intrinsic viscosity approaches to the real velocity measured with UVP. Therefore, it is worth remarking that the increased intrinsic viscosity reflecting the particle shape effects of concrete constituents should be essentially considered to predict the concrete flow in pipe. 
Table 3 Input values for the analysis of shear-induced particle migration.

\begin{tabular}{|c|c|}
\hline Parameters & Values \\
\hline \multicolumn{2}{|c|}{ Rheological properties of cement paste } \\
\hline Plastic viscosity (Pa s) & 1.0 \\
\hline Yield stress $(\mathrm{Pa})$ & 10.0 \\
\hline \multicolumn{2}{|c|}{ Rheological properties of mortar } \\
\hline Plastic viscosity (Pa s) & 2.5 \\
\hline Yield stress $(\mathrm{Pa})$ & 10.0 \\
\hline \multicolumn{2}{|c|}{ Maximum solid fraction } \\
\hline$\phi_{s, \max }$ & 0.675 \\
\hline$\phi_{g, \max }$ & 0.645 \\
\hline \multicolumn{2}{|c|}{ Volume fraction } \\
\hline$\phi_{s}$ & 0.458 \\
\hline$\phi_{g}$ & 0.349 \\
\hline \multicolumn{2}{|c|}{ Intrinsic viscosity } \\
\hline$\eta_{s}$ & 3.5 \\
\hline$\eta_{g}$ & 6.0 \\
\hline
\end{tabular}

$s$ and $g$ mean sand and aggregate, respectively.

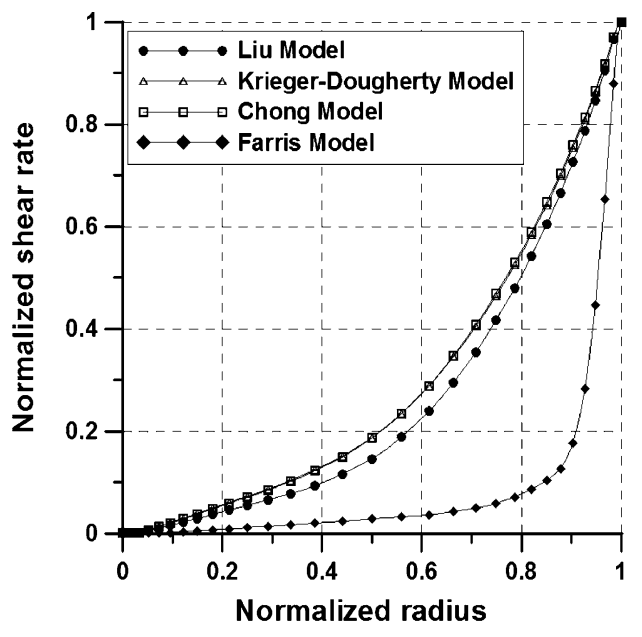

(a) Shear rate

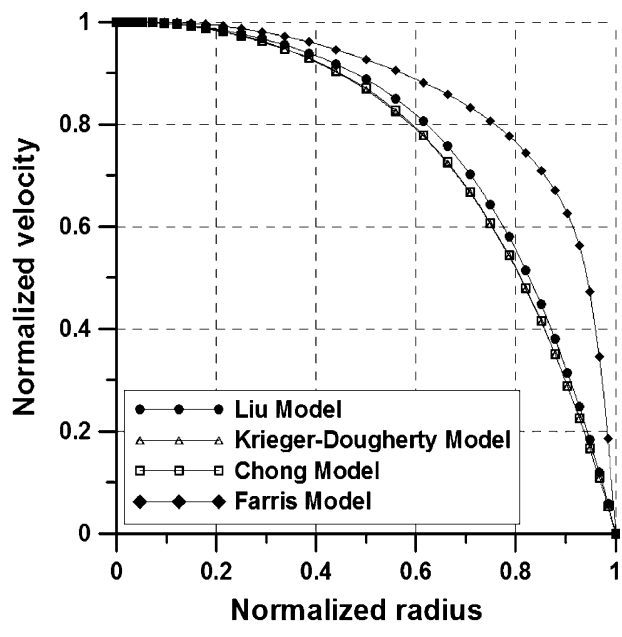

(b) Velocity

Fig. 7 Numerical simulation results of the shear-induced particle migration on the normalized shear rate and velocity profiles along the radius.

\subsection{Flow Rates}

The flow rates of concrete were compared with the actually measured flow rates of a $170 \mathrm{~m}$ full scale pumping test. The accuracy of the prediction was estimated with the following equation,

$$
\begin{aligned}
& \text { Accuracy }(\%) \\
& =\left(1-\frac{1}{n} \sum_{i=1}^{n} \frac{\mid \text { Measured flow rate }- \text { Predicted flow rate } \mid}{\mid \text { Measured flow rate } \mid}\right) \\
& \times 100
\end{aligned}
$$

In case of unimodal suspension models i.e. Liu model, Krieger-Dougherty model and Chong model, as shown in
Fig. 10, the calculated accuracy for the comparison made was 33, 35 and $37 \%$, respectively. However, in case of multimodal suspension model i.e. Farris model, without considering the particle shape effects, as shown in Fig. 11, the calculated accuracy become $82 \%$. When considering the particle shape effects with increased intrinsic viscosity in modified Farris model, the calculated accuracy goes up to $93 \%$, as shown in Fig. 12.

Through these comparison results, multimodal suspension approximation for concrete is right method to simulate the concrete flow in pipe and taking into account the particle shape effects would provide better insight to predict the pipe flow of pumped concrete. 


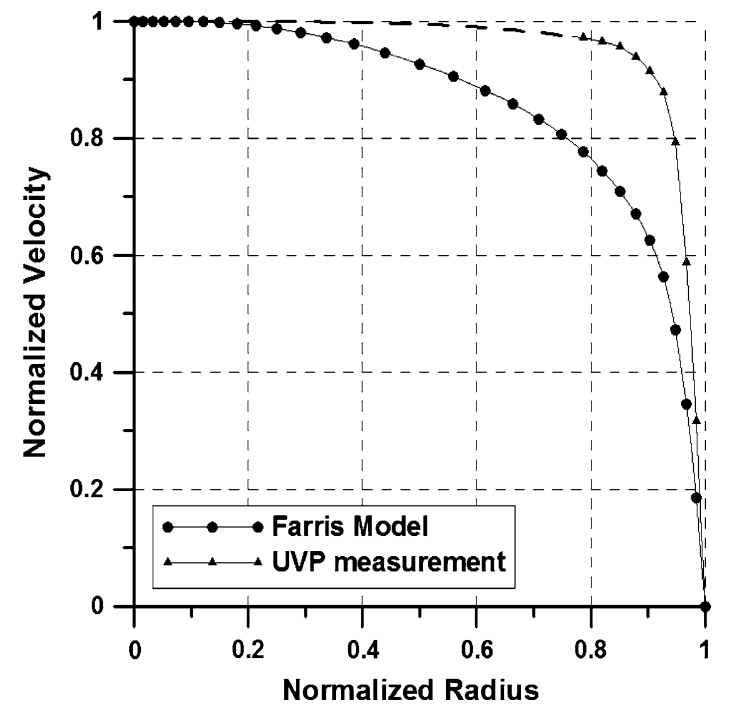

Fig. 8 Comparison of the normalized velocity profile along the radius between numerical simulation results using the multimodal suspension viscosity model (i.e. Farris model) and experimentally measured velocity profile using UVP.

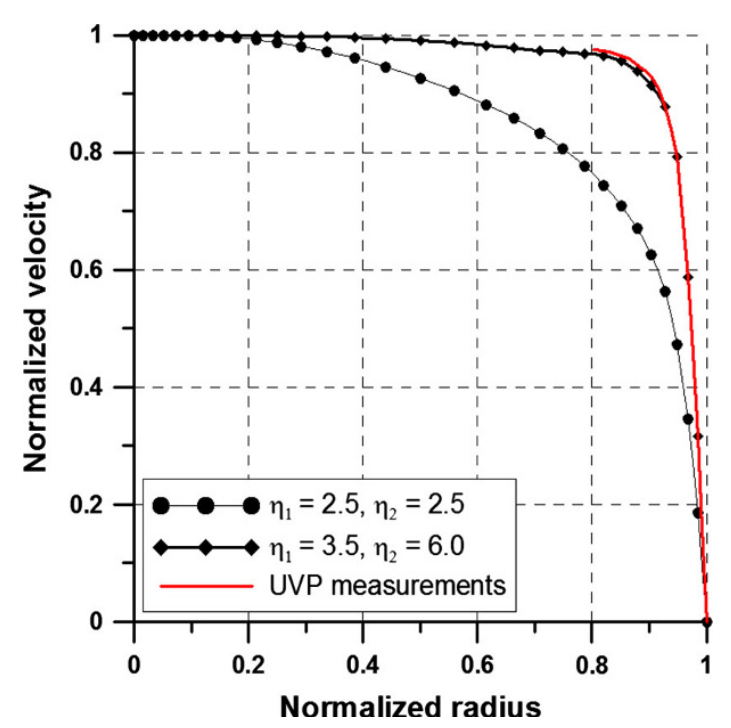

Fig. 9 Comparison of the normalized velocity profile along the radius between the numerical simulation results of with and without considering the particle shape effects and experimentally measured velocity profiles using UVP.

\section{Conclusion}

For the prediction of concrete pumping, numerical simulations considering four different viscosity models in the shear-induced particle migration analysis were conducted and calculated results were compared with the $170 \mathrm{~m}$ fullscale pumping tests in this study and the following conclusions were obtained.

1. When concrete is being pumped, the lubrication layer which is formed at the interface between the concrete and the wall of the pipe plays a dominant role to facilitate the concrete pumping.

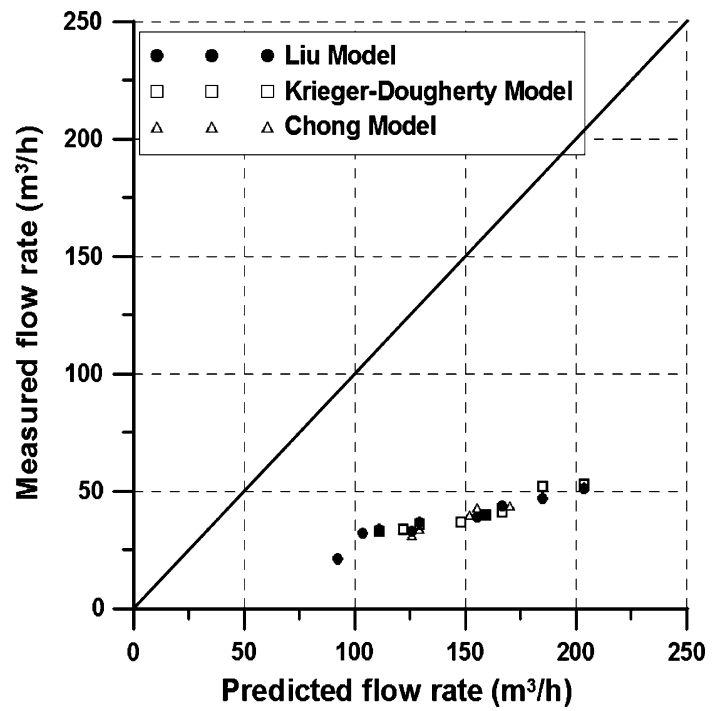

Fig. 10 Comparison of the flow rates between the numerically calculated results according to the three unimodal viscosity models and experimental data using a $170 \mathrm{~m}$ full scale pumping test.

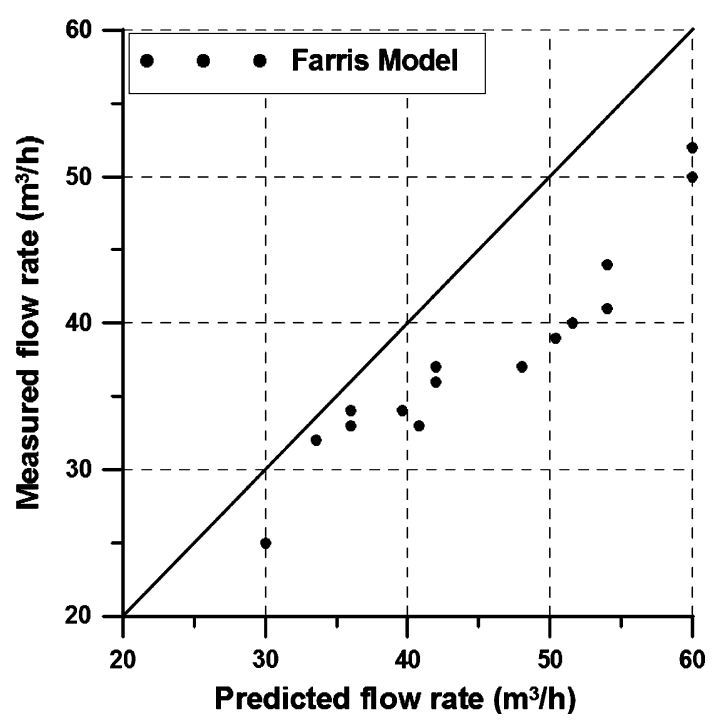

Fig. 11 Comparison of the flow rates between the multimodal viscosity model (i.e. Farris model) without considering the particle shape effects and experimental data using a $170 \mathrm{~m}$ full scale pumping test.

2. The shear-induced particle migration which demonstrated that particles in suspension migrate across the streamlines from a region of a higher shear rate to a region of a lower shear rate is a major possible mechanism that illustrates to the formation of the lubrication layer.

3. Regarding the shear-induced particle migration, the rheological parameters are a paramount factor to predict the pipe flow. Among four viscosity models used in this study, the multimodal viscosity model representing concrete as a three-phase material consisting of cement paste, sand and gravel can accurately simulate the lubrication layer and flow condition of pumped concrete. 


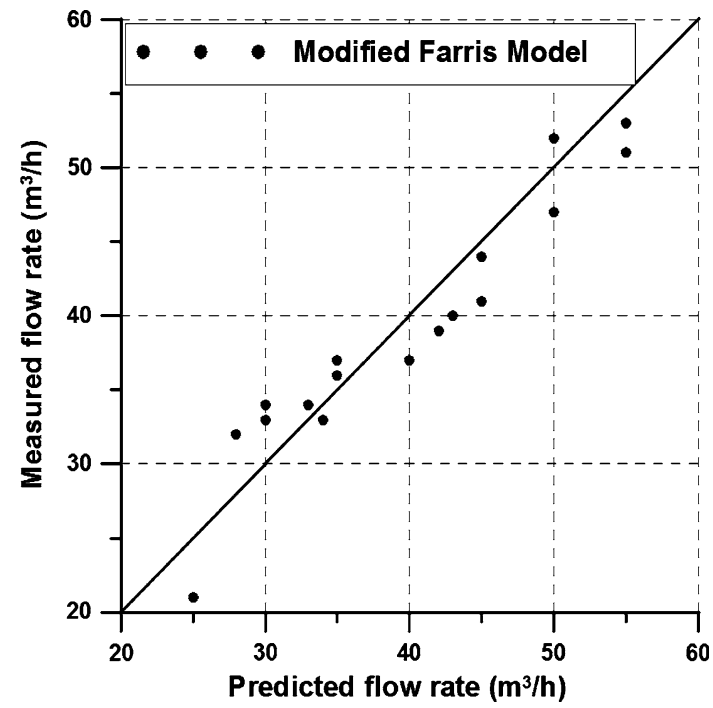

Fig. 12 Comparison of the flow rates between the multimodal viscosity model considering the particle shape effects and experimental data using a $170 \mathrm{~m}$ full scale pumping test.

4. When calculating the multimodal viscosity model, the increased intrinsic viscosity reflecting the particle shape effects of concrete constituents, especially the sand and the gravel should be considered to predict more exactly the real velocity profile and the flow rates of pumped concrete.

5. From this study, a comprehensive prediction methodology for the pipe flow of pumped concrete was established.

\section{Acknowledgments}

This research was supported by a Grant from the Construction Technology Innovation Program (08CTIPE01-Super Long Span Bridge R\&D Center) funded by Ministry of Land, Transportation and Maritime Affairs (MLTM) of Korean government.

\section{Open Access}

This article is distributed under the terms of the Creative Commons Attribution License which permits any use, distribution, and reproduction in any medium, provided the original author(s) and the source are credited.

\section{References}

Alekseev, S. N. (1952). On the calculation of resistance in pipe of concrete pumps. Mekhanizatia Storitel'stva, 9(1), 8-13. (Translated as Library Communication No. 450, Building Research Station, 1953).
Barnes, H. A., Hutton, J. F., \& Walters, K. (1989). An introduction to rheology. Amsterdam, Netherlands: Elsevier Science.

Browne, R. D., \& Bamforth, P. B. (1977). Tests to establish concrete pumpability. ACI Journal Proceedings, 74(5), 193-203.

Chailmo, T., Touloupov, N., \& Markovskiy, M. (1989). Peculiarities of concrete pumping. Minsk, Belarus: Stroikniga (In Russian).

Chateau, X., Ovarlez, G., \& Trung, K. L. (2008). Homogenization approach to the behavior of suspension of non-colloidal particles in yield stress fluids. Journal of Rheology, 52, 489-506.

Choi, M. S., Kim, Y. J., \& Kwon, S. H. (2013a). Prediction on pipe flow of pumped concrete base on shear-induced particle migration. Cement and Concrete Research, 52(10), 216-224.

Choi, M. S., Roussel, N., Kim, Y. J., \& Kim, J. K. (2013b). Lubrication layer properties during concrete pumping. Cement and Concrete Research, 45(3), 69-78.

Chong, J. S., Christiansen, E. B., \& Baer, A. D. (1971). Rheology of concentrated suspensions. Journal of Applied Polymer Science, 15, 2007-2021.

Ede, A. N. (1957). The resistance of concrete pumped through pipelines. Magazine of Concrete Research, 9(27), 129-140.

Farris, R. (1968). Prediction of the viscosity of multimodal suspensions from unimodal viscosity data. Transactions of the Society of Rheology, 12, 281-301.

Fluent Inc. (2011). User's guide FLUENT 13.0. Fluent, Pittsburgh.

Hafid, H., Ovarlez, G., Toussaint, F., Jezequel, P. H., \& Roussel, N. (2010). Estimating measurement artifacts in concrete rheometers from MRI measurements on model materials. In Proceedings of SCC2010, Montreal, Canada, pp. 127-137.

Jacobsen, S., Haugan, L., Hammer, T. A., \& Kalogiannidis, E. (2009). Flow conditions of fresh mortar and concrete in different pipes. Cement and Concrete Research, 39(11), 997-1006.

Jo, S. D., Park, C. K., Jeong, J. H., Lee, S. H., \& Kwon, S. H. (2012). A computational approach to estimating a lubricating layer in concrete pumping. Computers Materials and Continua, 27(3), 189-210.

Kaplan, D., De Larard, F., \& Sedran, T. (2005). Design of concrete pumping circuit. ACI Materials Journal, 102(2), 110-117.

Koehler, E. P., Fowler, D. W., Ferraris, C. F., \& Amziane, S. (2006). A new portable rheometer for fresh self consolidating concrete. ACI Special Publication, 233, 97-116.

Krieger, I. M., \& Dougherty, T. J. (1959). A mechanism for non-newtonian flow in suspensions of rigid spheres. Transactions of the Society of Rheology, 3, 137-152.

Kwan, A. K. H., Mora, C. F., \& Chan, H. C. (1999). Particle shape analysis of coarse aggregate using digital image processing. Cement and Concrete Research, 29, 1403-1410.

Kwon, S. H., Jo, S. D., Park, C. K., Jeong, J. H., \& Lee, S. H. (2013). Prediction of concrete pumping: Part I. 
Measurements of rheological properties of lubricating layer. ACI Materials Journal, 110(6), 647-656.

Lachemi, P. M., Hossain, K. M. A., Lambros, V., Nkinamubanzi, P. C., \& Bouzoubaa, N. (2004). Performance of new viscosity modifying admixtures in enhancing the rheological properties of cement paste. Cement and Concrete Research, 34, 185-193.

Leighton, D., \& Acrivos, A. (1987a). The shear-induced selfdiffusion in concentrated suspensions. Journal of Fluid Mechanics, 181, 415-439.

Leighton, D., \& Acrivos, A. (1987b). Measurement of shearinduced self-diffusion in concentrated suspensions of Spheres. Journal of Fluid Mechanics, 177, 109-131.

Liu, D. M. (2000). Particle packing and rheological property of highly-concentrated ceramic suspensions: Determination and viscosity prediction. Journal of Materials Science, 35, 5503-5507.

Met-flow, S. A. (2002). Model UVP-duo with software version 3 user's guide. Switzerland: Met-flow Co.

Mitschka, P. (1982). Simple conversion of Brookfield R.V.T readings into viscosity functions. Rheologica Acta, 21, 207-209.

Mora, C. F., \& Kwan, A. K. H. (2000). Sphericity, shape factor, and convexity measurement of coarse aggregate for concrete using digital image processing. Cement and Concrete Research, 30, 351-358.
Morinaga, S. (1973). Pumpability of concrete and pumping pressure in pipelines. Proceedings of Rilem Seminar, Leeds, 3, 1-39.

Otsu, N. A. (1979). Threshold selection method from gray level histogram. IEEE Trans on Systems, Man and Cybernetics, 9(1), 62-69.

Phillips, R. J., Armstrong, R. C., Brown, R. A., Graham, A. L., \& Abbot, J. R. (1992). A constitutive equation for concentrated suspensions that accounts for shear-induced particle migration. Physics of Fluids, 4, 30-40.

Sakuta, M., Kasanu, I., Yamane, S., \& Sakamoto, A. (1989). Pumpability of fresh concrete. Tokyo, Japan: Takenaka Technical Research Laboratory.

Struble, L., \& Sun, G. K. (1995). Viscosity of Portland cement paste as a function of concentration. Advanced Cement Based Materials, 2(2), 62-69.

Szecsy, R. S. (1997). Concrete rheology. Ph.D thesis, University of Illinois, Urbana-Champaign, IL.

Tanigawa, Y., Mori, H., \& Noda, Y. (1991). Theoretical study on pumping of fresh concrete. Concrete Institute of Japan.

Wallevik, J. E. (2008). Minimizing end-effects in the coaxial cylinders viscometer: Viscoplastic flow inside the ConTec BML viscometer 3. Journal of Non-Newtonian Fluid Mechanics, 155(3), 116-123.

Weber, R. (1968). The transport of concrete by pipeline. London, UK: Cement and Concrete Association. 